\title{
Gravity is the Solution for World Energy Crises
}

\section{Nikolayenko Al*}

210-9380 Lajeunesse, Montreal, QC H2M 1S4, Canada

\section{The Concept of this Invention is Employment of a Downwards Moving Object to Produce Electricity}

There is nothing new in this idea, and as everyone before me, I encountered the same problem: lifting the object takes far more energy.

To resolve the problem, I took a different approach and employed balance, leverage and counterbalances as shown on the (Figure 1). The large circle represents the downward moving object or balance smaller circles represent counterbalances (combined mass of both counterbalances equal to the mass of the balance), black triangles represent fulcrums, and lines connecting centers of the circles represent levers. The leverage ratio is $1: 2$. Due to this ratio, when balance is moving downwards, only half of the energy is used to lift the counterbalances.

But not so fast if we try to flip the system around axis $\mathrm{X}$ and repeat the move, we will soon discover that the side where balance is located is heavier than the side with the counterbalances; all energy gain will be lost on flipping. It is obvious on the Figure 1 that the center of the large circle is twice farther from rotation axis $\mathrm{X}$ than centers of both counterbalances.

On July 17, 2014, a solution of the problem came unexpectedly, and I replaced the middle parts of the two levers with a leaf spring (Figure 2). As a result, balance shifted downwards lifting both counterbalances

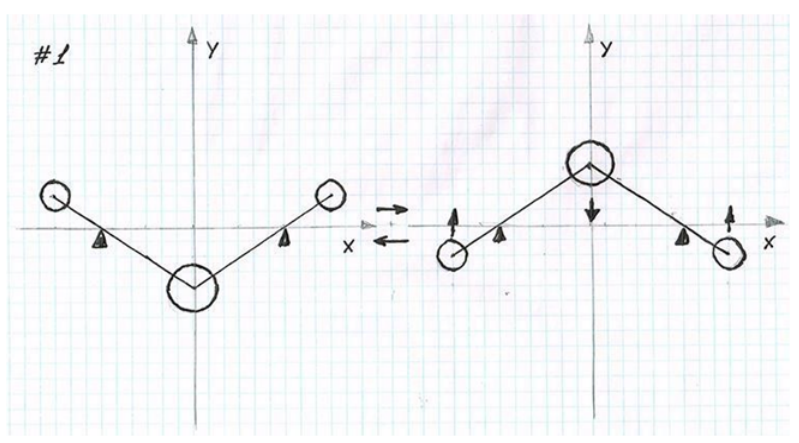

Figure 1: Leverage and counterbalances.

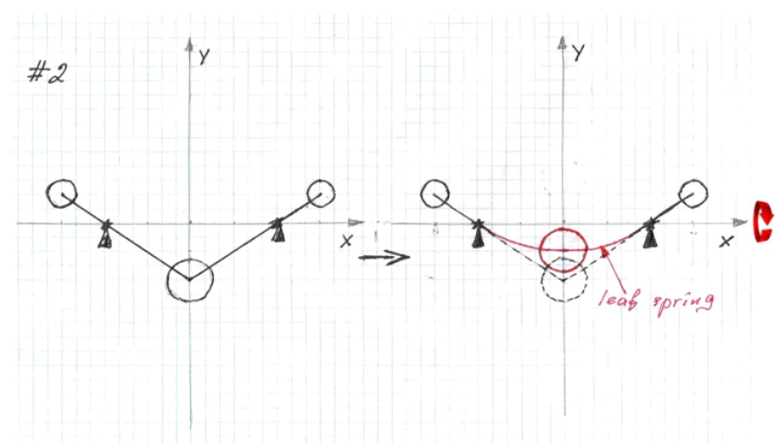

Figure 2: Levers with a leaf Spring. and the entire system kept its equilibrium. Later I named it 'compound leverage'. Keep in mind, the system does not induce its rotation a small motor is needed.

Replacing the balance (middle piece) with a linear generator allows gravity-to-electricity conversion. Due to system equilibrium, only a small portion of that energy is spent on rotation.

This Device has no Conflict with Newtonian Law; it Transforms $\mathbf{1} / 3$ of Gravitational Force Applied to Linear Generator (Middle Piece) into Ready-To-Consume Electricity

Do not embarrass yourself by denying, that the described system insures the balance downward movement, lifts counterbalances using only half of that energy and maintains equilibrium if rotated around axis X.

Let's take a closer look. On Figure 3, the system is depicted from a different point of view (Axis X is pointing directly at your eye). Also, a pendulum is attached.

1. Pendulum and its trajectory

2. Counterbalances (second is hidden behind)

3. Point of 180 degree rotation (axis $\mathrm{x}$ as on Figures 1 and 2 are directed towards you)

4. Compound leverage

5. Balance

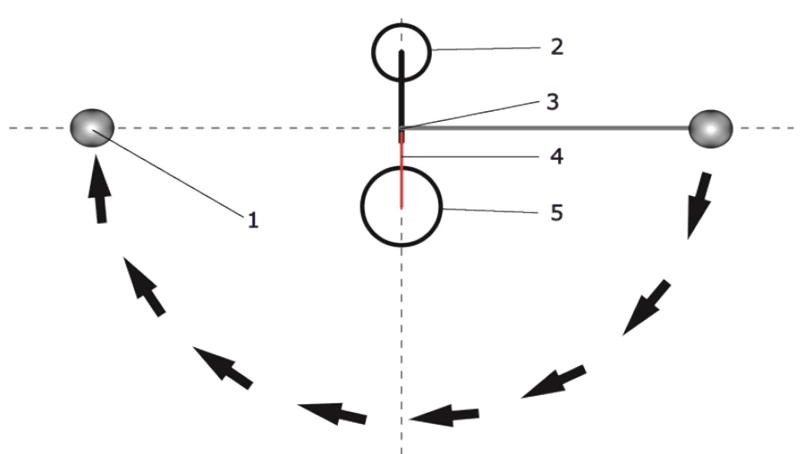

Figure 3: Pendulum is attached.

*Corresponding author: Nikolayenko Al, 210-9380 Lajeunesse, Montreal, QC H2M 1S4, Canada, Tel: 514-577-9593; E-mail: anatoly@videotron.ca

Received October 31, 2015; Accepted November 18, 2015; Published November 28,2015

Citation: Nikolayenko Al (2015) Gravity is the Solution for World Energy Crises. $J$ Appl Mech Eng 4: 185. doi:10.4172/2168-9873.1000185

Copyright: ( 2015 Nikolayenko Al, et al. This is an open-access article distributed under the terms of the Creative Commons Attribution License, which permits unrestricted use, distribution, and reproduction in any medium, provided the original author and source are credited. 
Suppose, pendulum does not move until the balance reached its lowest point conversely, balance does not move until the pendulum reached its highest point on the other side, also friction in bearings supporting system rotation and aerodynamic resistance do not exist. What will happen then? The pendulum will not stop, the system will move forever and the generator will not stop producing electricity, aka 'perpetual motion'.

Now back to reality. The ideal condition is movement without friction (but this does not exist). But, we can reduce friction to a minimum. Also, an increase of balance/counterbalance mass has a negligible impact on a pendulum's movement. This means that electricity produced by a generator weighing $1 \mathrm{~kg}$ is not enough to sustain its rotation. A generator weighing 100 pounds will produce enough power to rotate itself forever, and a generator weighing 10 tons will produce electricity for your entire neighborhood. Need more? Make it heavier.

\section{Intriguing? Let's Go Further}

Previously, I described the simplest model. But what if rotation and reciprocal balance movement are happening at the same time? It might produce some new force obstructing system rotation to the point of making the entire invention useless.

First, separation of two movements using a pendulum as previously described will eliminate such force.

Second, yes, centrifugal force shall be taken into account building this machine. Look at the Figure 4; the system is rotating around axis X. In the situation shown on Figure $4 \mathrm{a}$, the balance and counterbalances are moving towards axis $\mathrm{X}$ until they reach it centrifugal force opposes this move, but past the axis X Figure $4 \mathrm{~b}$, 'au contraire', the centrifugal force enforces the move. Inother words, in a certain period of the rotation, we are losing energy due to centrifugal force; in the other period, we are gaining it back. Total gain/loss of energy is equal to zero.

There is another effect that affects system performance. I call it the 'figure skater effect'. When a figure skater is spinning, extending his arms slows him down contrary to pulling his arms as close as possible, which accelerates his rotation. Similar to centrifugal effect, it happens during coincident rotation and back-and-forth movements.

Now take a look at Figure 4a again. The figure skater is pulling his arms, it accelerates him. Figure $4 \mathrm{~b}$, the figure skater extending his arms, it slows him down. Here again, in one period we are losing energy in another we are gaining energy back.

As you might notice in the situation depicted on Figure 4a, we are losing momentum due to centrifugal force but gaining it due to figure skater effect on Figure $4 \mathrm{~b}$ a reverse situation, centrifugal force is giving back the figure skater's taking away. Both of the forces supposedly impeding the Gravity Powered Generator are compensating for each other bringing their combined result to zero. Combined zero hindering is exactly what is needed.
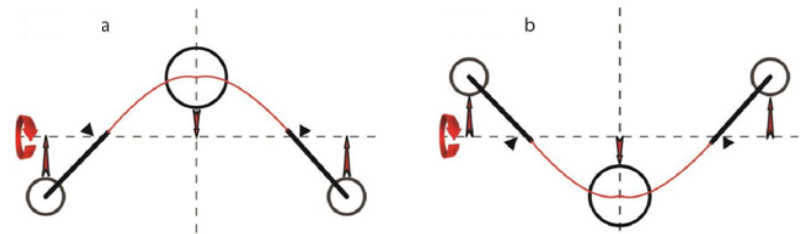

Figure 4: $(a, b)$. Figure skater extending his arms and Figure skater's taking away.

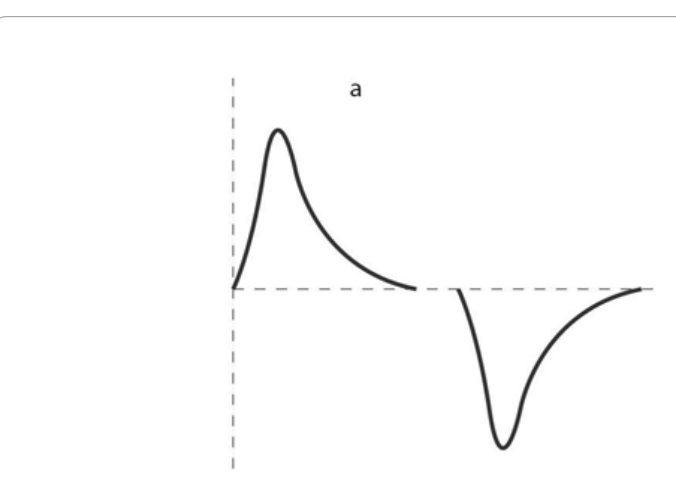

Figure 5a: Displayed electric current produced.

b

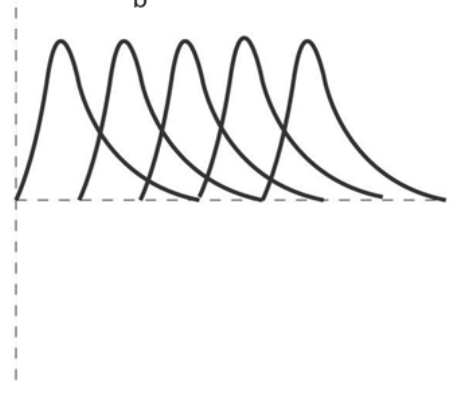

Figure 5b: Direct electric current produced.

Fully loaded generator makes one full rotation in 5-10 seconds. Similar to conventional generator, increased demand for electricity from generator creates more resistance to its move and consequently to system rotation, it translates into lesser speed. Lesser speed means less figure skater and centrifugal effects. Generator working on the upper limit of its capacity also shows maximum efficiency.

Forget for a moment what modern inquisition says. If every piece of the puzzle is falling into place, what does common sense tell you?

\section{What Kind of Electricity we are Getting?}

Aiming to reduce the friction, a conventional generator cannot be used; a linear DC generator is an obvious choice. Full rotation of the machine takes between 5 and 10 seconds to complete. During this time the generator performs one complete back-and-forth movement. On Figure $5 \mathrm{a}$ is a displayed electric current produced by such movement. The graphic is similar to alternate current but 250-600 times slower. Also, the graphic shows interruption in electric current caused by a period between the generator's full stop and resuming its movement in another direction; depending on apparatus design, this interruption can last from a 'fraction of a heartbeat' or, like in the pendulum's case, up to 3 seconds.

We can easily flip the low part of the graphic upwards using a rectifier or generator brushes, etc. Then by interconnecting several generators on a different rotation period, we will get a direct current, see Figure 5b. It sounds complicated, but your computer like most electrical devices around you takes AC and transforms it into DC; there is no alternate current inside it. Chemical plants, smelters, steel mills, railways, and subways consume mostly DC. 


\section{Can i buy this Generator for my Home?}

Since the Canadian government refused to patent this invention, further development stopped. It will take some more Chernobyls and Fukushimas before you will be able to install this generator in your basement.

\section{Can it be used on Road Vehicles?}

Yes, after a slight modification my invention will replace the internal combustion engine. What kind of modification? Well this "little" secret I am taking with me.

*In this article: balance, large circle, or linear generator all mean the same.

${ }^{* *}$ Extending the distance from fulcrums to counterbalances will reduce the mass of the latter still maintaining equilibrium. Such modification shall not increase power or efficiency of the machine but decrease its total weight.

(C) Images and text of this article ARE NOT copyright protected. 